Pacific Journal of Mathematics

APPROXIMATE IDENTITIES FOR CONVOLUTION MEASURE 


\title{
APPROXIMATE IDENTITIES FOR CONVOLUTION MEASURE ALGEBRAS
}

\author{
Charles D. LAHR
}

Let $(A, *)$ be a commutative semisimple convolution measure algebra with structure semigroup $\Gamma$. It is proved that $A$ has a weak bounded approximate identity if and only if $\Gamma$ has a finite set of relative units; moreover, $\Gamma$ has an identity if and only if some weak bounded approximate identity is of norm one. Considering now a commutative semigroup $S$, the existence of a bounded (norm) approximate identity in $A=\ell_{1}(S)$ is equivalent to the existence in $S$ of a finite number of nets $\left\{u_{\rho(i)}\right\}_{\rho(i) \in F_{i}}, i=1,2, \cdots, n$ with the property that for every $x \in S$ there exist $j$ and $\rho(j)_{x}$ such that $\rho(j) \geqq \rho(j)_{x}$ implies $x u_{\rho(j)}=x$.

Basic definitions, notation, and background results are given in $\S 2$. In $\S 3$ we study the relationship between $\left(A,{ }^{*}\right)$ and $\Gamma$ when $A$ has a weak bounded approximate identity of norm $R$. In $\S 4$ we examine conditions on $S$ that are equivalent to the existence of a bounded (norm) approximate identity in $A=\ell_{1}(S)$.

2. Preliminaries. Let $\left(B,{ }^{*}\right)$ be a commutative Banach algebra under $\|\cdot\|$. Let $\Delta(B)$ denote the maximal ideal space of $B$, that is, the space of all continuous homomorphisms of $B$ into the complex field $C$ together with the weak-*(Gelfand) topology [9]. As usual for any $\alpha \in B$, define $\hat{\alpha}(\chi)=\chi(\alpha)$ for each $\chi \in \Delta(B)$, and let

$$
\hat{B}=\{\hat{\alpha}: \alpha \in B\} \text {. }
$$

A weak bounded approximate identity of norm $R$ for $B$ is a net $\left\{E_{\rho}\right\}_{\rho \in \mathscr{F}}$ of elements of $B$ such that (a) $\left\|E_{\rho}\right\| \leqq R$ for some positive number $R$ and for all $\rho \in \mathscr{F}$, and (b) $\left(\alpha^{*} E_{\rho}\right)^{\wedge}(\chi) \rightarrow \hat{\alpha}(\chi)$ for all $\chi \in \Delta(B)$ and for every $\alpha \in B$. A bounded approximate identity of norm $R$ for $B$ is a net $\left\{E_{\rho}\right\}$ of elements of $B$ such that (a) $\left\|E_{\rho}\right\| \leqq R$ for some positive number $R$ and for all $\rho$, and (b) $\left\|\alpha^{*} E_{\rho}-\alpha\right\| \rightarrow 0$ for all $\alpha \in B$; we sometimes use the terminology "bounded (norm) approximate identity of norm $R$ " for the same concept.

If $X$ is any normed linear vector space, the continuous linear dual of $X$ is denoted by $X^{*} ;(\alpha, f)$ represents the action of $f \in X^{*}$ on $\alpha \in X$; and if $Y \subseteq X, Z \subseteq X^{*}$, then let $w(Y, Z)$ be the weak topology on $Y \subseteq X$ induced by $Z \subseteq X^{*}$. The natural mapping of $X$ into $X^{* *}$ is denoted by $j: X \rightarrow X^{* *}$. Often, we simply denote $(f, j(\alpha))$ by $(\alpha, f), \alpha \in X, f \in X^{*}$, in those circumstances where the 
meaning is clear.

Now let $S$ be a commutative locally compact Hausdorff semigroup with jointly continuous multiplication (sometimes herein referred to as a commutative locally compact topological semigroup); and let $M(S)$ denote the complex Banach algebra of all bounded regular Borel measures on $S$ where the product ${ }^{*}$ is defined by convolution. For $\mu, \nu \in M(S), F$ a Borel subset of $S$,

$$
\left(\mu^{*} \nu\right)(F)=\int_{S} \int_{S} \dot{\phi}_{F}(x y) d \mu(x) d \nu(y),
$$

where $\phi_{F}$ denotes the characteristic function of $F$. The norm on $M(S)$ is the total variation norm, denoted $\|\cdot\|$. See Taylor [10]. A semicharacter $\chi$ on $S$ is a nonzero continuous complex valued function on $S$ of modulus less than or equal to one which satisfies

$$
\chi(x y)=\chi(x) \chi(y) \quad \text { for all } x, y \in S \text {. }
$$

The collection of semicharacters of $S$ is denoted by $\hat{S}$. It is well known that $C_{0}(S)^{*}=M(S)$, where $\mu \in M(S)$ induces a linear functional on $C_{0}(S)$ by

$$
(g, \mu)=\int_{S} g(x) d \mu(x) \quad \text { for all } g \in C_{0}(S) .
$$

This paper is devoted to characterizations of approximate identities for certain semisimple convolution measure algebras. For a definition of convolution measure algebra see [10]. If $G$ is a locally compact abelian topological group, and if $L_{1}(G)$ is the algebra of Haar integrable functions on $G$ under convolution multiplication, then $L_{1}(G)$ is a convolution measure algebra. Likewise, $M(S)$ is a convolution measure algebra.

Taylor proves in [10] that if $(A, *)$ is a commutative convolution measure algebra, we may identify the maximal ideal space of $\left(A,{ }^{*}\right)$ with $\hat{\Gamma}$, the set of all semicharacters on a compact topological semigroup $\Gamma$, which he labels the structure semigroup of $\left(A,{ }^{*}\right.$ ), (we will denote the structure semigroup of any convolution measure algebra under discussion by $\Gamma$ ). There is a homomorphism $p: \mu \rightarrow \mu_{p}$ of $A$ into $M(\Gamma)$ with the following pertinent properties: $p(A)$ is weak-* dense in $M(\Gamma)$, that is, dense in the $w(M(\Gamma), C(\Gamma))$ topology (where we have identified $C(\Gamma)$ with its natural image in $\left.M(\Gamma)^{*}\right) ; p$ is an isometry if and only if $\left(A,{ }^{*}\right)$ is semisimple. $\Gamma$ also has the property that the uniformly closed linear span of $\hat{\Gamma}$ is $C(\Gamma)$. We make use of this fact in observing that $\hat{\Gamma} \cong \Delta(M(\Gamma))$ is enough to imply the semisimplicity of $M(\Gamma)$ : that is, suppose $\mu, \nu \in M(\Gamma)$ and $\hat{\mu}(\chi)=\hat{\nu}(\chi)$ for all $\chi \in \hat{\Gamma}$; then because the linear span of $\hat{\Gamma}$ is uniformly dense 
in $C(\Gamma)$, the formula $(\chi, \mu)=(\chi, \nu)$ for $\chi \in \hat{\Gamma}$ can be extended to all $f \in C(\Gamma)$; therefore, $\mu$ and $\nu$ agree as linear functionals on $C(\Gamma)$ and so as elements of $M(\Gamma)$.

The set of discrete measures in $M(S)$ forms a subalgebra of $M(S)$, denoted by $\ell_{1}(S)$. Of course, if $S$ is discrete, then $\ell_{1}(S)=$ $M(S)$. Hewitt and Zuckerman present a detailed study of $\ell_{1}(S)$ in [5]. An interesting fact is that the existence of an identity in $\ell_{1}(S)$ is equivalent to the existence of a finite set of relative units in $S$, where $U$ is defined to be a set of relative units for $S$, if for every $x \in S$, there exists $u \in U$ such that $x u=x$. Lardy [6] proves that the same conditions on $S$ are necessary and sufficient for the existence of an identity in $M(S)$, and in fact an identity for $M(S)$ must lie in $\ell_{1}(S)$.

3. Weak approximate identities. Throughout this section $\left(A,{ }^{*}\right)$ is a semisimple convolution measure algebra. Assume that $A$ is embedded in $M(\Gamma)$. The first result relates the existence of a weak bounded approximate identity in $A$ to the existence of an identity in $M(\Gamma)$.

THEOREM 3.1. $\Gamma$ has a finite set of relative units if and only if $A$ has a weak bounded approximate identity.

Proof. Suppose $A$ has a weak bounded approximate identity $\left\{E_{\rho}\right\},\left\|E_{\rho}\right\| \leqq R$ for all $\rho$. Then $\left\{E_{\rho}\right\}$ is a subset of the closed ball of $M(\Gamma)$ of radius $R$. In the weak-* topology, this ball is compact. Thus, there is a subnet $\left\{E_{\rho^{\prime}}\right\}$ and $E \in M(\Gamma)$ such that $E_{\rho^{\prime}} \rightarrow E$ in the weak-* topology of $M(\Gamma)$. Our aim is to show that $E$ is an identity for $M(\Gamma)$. If $\alpha \in A,\left(\chi, \alpha^{*} E_{\rho}\right) \rightarrow(\chi, \alpha)$ for all $\chi \in \hat{\Gamma}$. Thus, $\left(\chi, \alpha^{*} E\right)=(\chi, \alpha)$ for all $x \in \hat{\Gamma}$ implies that $\alpha^{*} E=\alpha$ for all $\alpha \in A$. Since $A$ is weak-* dense in $M(\Gamma)$ [10], if $\mu$ is an arbitrary element of $M(\Gamma)$, there exists a net $\left\{\mu_{d}\right\} \subset A$ such that $\mu_{d} \rightarrow \mu$ in the weak-* topology; hence, $\left(\chi, \mu_{d}^{*} E\right) \rightarrow\left(\chi, \mu^{*} E\right)$ for all $\chi \in \hat{\Gamma}$, while $\left(\chi, \mu_{d}^{*} E\right)=\left(\chi, \mu_{d}\right) \rightarrow(\chi, \mu)$ for all $\chi \in \hat{\Gamma}$. Therefore, $\left(\chi, \mu^{*} E\right)=$ $(\chi, \mu)$ for all $\chi \in \hat{\Gamma}$ implies that $\mu^{*} E=\mu$ for all $\mu \in M(\Gamma)$, and hence $E$ is an identity for $M(\Gamma)$. Finally, $\Gamma$ has a finite set of relative units [6].

Conversely, assume now that $\Gamma$ has a finite set of relative units and without loss of generality assume that $U=\left\{\gamma_{1}, \gamma_{2}, \cdots, \gamma_{n}\right\}$ is a minimal set of relative units with $\gamma_{i}^{2}=\gamma_{i}$ for all $i$ [6]. Let $\left\{\omega_{\rho(i)}\right\}_{\rho(i) \in \mathscr{F}_{i}}$ be a neighborhood system for $\gamma_{i}, i=1,2, \cdots, n$; for each $i, \mathscr{F}_{i}$ is ordered by set inclusion, that is, $\rho(i) \geqq \rho^{\prime}(i)$ if and only if 
$\omega_{\rho(i)} \subseteq \omega_{\rho^{\prime}(i)}$. Now, since $A$ is a weak-* dense $L$-subspace of $M(\Gamma)$, for each $\rho(i) \in \mathscr{F}_{i}$, there is a nonnegative measure $\mu_{\rho(i)}$ of norm one in $A$ such that $\mu_{\rho(i)}$ is concentrated on $\omega_{\rho(i)}, i=1,2, \cdots, n$ [10]. For each $f \in C(\Gamma)$, we assert that $\left(f, \mu_{\rho(i)}\right) \rightarrow\left(f, \delta_{\gamma_{i}}\right), i=1,2, \cdots, n$ : fix $i$ and let $\varepsilon>0$ be given; there exists $\rho_{0}(i)$ such that

$$
\left|f(\gamma)-f\left(\gamma_{i}\right)\right|<\varepsilon \quad \text { for all } \gamma \in \omega_{\rho_{0}(i)}
$$

by continuity of $f$; thus, $\rho(i) \geqq \rho_{0}(i)$ implies

$$
\begin{aligned}
\left|\int_{\Gamma} f(\gamma) d \mu_{\rho(i)}(\gamma)-f\left(\gamma_{i}\right)\right| & =\left|\int_{\Gamma}\left[f(\gamma)-f\left(\gamma_{i}\right)\right] d \mu_{\rho(i)}(\gamma)\right| \\
& \leqq \int_{\omega_{\rho(i)}}\left|f(\gamma)-f\left(\gamma_{i}\right)\right| d \mu_{\rho(i)}(\gamma)<\varepsilon .
\end{aligned}
$$

Further, by the weak-* joint continuity of convolution multiplication on the unit ball of $M(\Gamma)[4]$, for $(\rho(i), \rho(j)) \in \mathscr{F}_{i} \times \mathscr{F}_{j}$

$$
\mu_{\rho_{(i)}} * \mu_{\rho(j)} \longrightarrow \delta_{r_{i}} * \delta_{r_{j}}=\delta_{r_{i} r_{j}}
$$

weak-* for all $i$ and $j=1,2, \cdots, n, i \neq j$.

Now, set $\mathscr{F}=\mathscr{F}_{1} \times \mathscr{F}_{2} \times \cdots \times \mathscr{F}_{n}$ and let $\rho \in \mathscr{F}$ be denoted by $\rho=(\rho(1), \rho(2), \cdots, \rho(n))$; for each $\rho \in \mathscr{F}$ define

$$
\begin{aligned}
E_{\rho}= & \sum_{1 \leq i \leqq n} \mu_{\rho(i)}-\sum_{1 \leqq i<j \leqq n} \mu_{\rho(i)} * \mu_{\rho(j)} \\
& +\cdots+(-1)^{n+1} \mu_{\rho(1)} * \mu_{\rho(2)} * \ldots * \mu_{\rho(n)} .
\end{aligned}
$$

If $E$ is the Hewitt-Zuckerman identity for $M(\Gamma)$ constructed from $U$ (see [5] and [6]), then from above $\left(\chi, E_{\rho}\right) \rightarrow(\chi, E)$ for each $\chi \in \hat{\Gamma}$, which implies $\left(\chi, \mu^{*} E_{\rho}\right) \rightarrow\left(\chi, \mu^{*} E\right)=(\chi, \mu)$ for each $\chi \in \hat{\Gamma}$ and for all $\mu \in A$. Clearly, $\left\|E_{\rho}\right\| \leqq R$ for some positive number $R$ and for all $\rho$. Thus, $\left\{E_{\rho}\right\}$ is a weak bounded approximate identity for $A$. This completes the proof.

The next corollary should be contrasted with Theorem 3.1 of [10]. We are unable to verify that the existence of an identity in $\Gamma$ implies the existence of a bounded approximate identity in $A$ of norm one.

Corollary 3.2. $\Gamma$ has an identity if and only if $A$ has a weak bounded approximate identity $\left\{E_{\rho}\right\}$ of norm one.

Proof. If $\Gamma$ has an identity $e$, let $\left\{\omega_{\rho}\right\}$ be a neighborhood system at $e$. Since $A$ is a weak-* dense $L$-subspace of $M(\Gamma)$, there is a nonnegative measure $E_{\rho}$ of norm one in $A$ such that $E_{p}$ is concentrated on each $\omega_{\rho}$. As in the proof of Theorem 3.1 it is seen that 
$\left\{E_{\rho}\right\}$ is a weak bounded approximate identity for $A$ since

$$
\left(\chi, \mu^{*} E_{\rho}\right) \longrightarrow\left(\chi, \mu^{*} \delta_{e}\right)=(\chi, \mu)
$$

for all $\chi \in \hat{\Gamma}$ and for each $\mu \in A$.

Conversely, if $\left\{E_{\rho}\right\}$ is a weak bounded approximate identity of norm one, the proof of Theorem 3.1 shows that there is a subnet $\left\{E_{\rho^{\prime}}\right\}$ and $E \in M(\Gamma)$ such that $E_{\rho}, \rightarrow E$ in the weak-* topology and $E$ is the identity of $M(\Gamma)$. In fact, $E$ is the Hewitt-Zuckerman identity for $\ell_{1}(\Gamma)$ formed from the set of relative units of $\Gamma$ [6]. Thus, $\left\|E_{\rho}\right\| \leqq 1$ for all $\rho$ implies $\|E\|=1$, so $E$ is concentrated on a single point $e$ of $\Gamma$. Thus, $e$ is the identity of $\Gamma$.

COROLlaRy. 3.3. A has a weak bounded approximate identity if and only if $M(\Gamma)$ has an identity.

Proof. $M(\Gamma)$ has an identity if and only if $\Gamma$ has a finite set of relative units [6].

4. Approximate identities for $\ell_{1}(S)$. We now begin the study of $\ell_{1}(S)$. The semisimplicity of $\ell_{1}(S)$ is equivalent to the algebraic condition on $S$ that $x^{2}=y^{2}=x y$ implies $x=y, x, y \in S$ [5]. If $S$ satisfies this condition, we follow Petrich [7, p. 311] in saying that $S$ is separative.

The maximal ideal space of $\ell_{1}(S)$ is just $\hat{S}$. If $\Lambda$ is the uniformly closed subspace of $\ell_{1}(S)^{*}=\ell_{\infty}(S)$ generated by $\hat{S}$, then $A$ is a $C^{*}$-algebra with identity. Therefore, $\Gamma$, the maximal ideal space of $\Lambda$, is compact and if $\hat{S}$ separates points of $S$, then there is a natural isomorphism $i_{s}$ of $S$ onto a dense subset of $\Gamma$. Also note that $\left(\chi, i_{s}(x)\right)=\chi(x)$ for all $x \in S, \chi \in \hat{S}$. The author originally proved all of these results by pursuing Rennison's techniques [8] of applying the Arens product [1] to the task of describing $\Delta\left(\ell_{1}(S)\right)$. It was very kind of the referee to point out the much simpler arguments just presented. We restate the main conclusion in the next proposition.

Proposition 4.1. If $S$ is separative, then there is a natural isomorphism $i_{s}$ of $S$ onto a dense subset of $\Gamma$.

In examining conditions on $S$ that are imposed by the existence of a norm approximate identity in $\ell_{1}(S)$, we find that the following proposition holds without regard to the semisimplicity of $\ell_{1}(S)$.

Proposition 4.2. Let $S$ be a commutative semigroup. If $\ell_{1}(S)$ 
has a norm approximate identity $\left\{E_{\rho}\right\}$ (bounded or not), then $S$ has $a$ set of relative units.

Proof. Let $x \in S$. Then there exists $\rho$ such that

$$
\left\|\delta_{x} * E_{\rho}-\delta_{x}\right\|<\frac{1}{4}
$$

Set $E_{\rho}=\sum_{i=1}^{\infty} \varepsilon_{i} \delta_{g_{i}} . \quad$ Then

$$
\left|1-\sum_{\substack{i \\ g_{i}=x}} \varepsilon_{i}\right|<\frac{1}{4}
$$

implies there exists $g \in S$ such that $g x=x$.

COROLLARY 4.3. If $S$ is a cancellative semigroup and if $\ell_{1}(S)$ has a norm approximate identity, then $S$ has an identity.

Proof. Let $x \in S$. Then there exists $z \in S$ such that $x z=x$. Now if $y \in S, x z y=x y$ implies $z y=y$, so $z$ is the identity for $S$.

Proposition 4.4. Suppose $\left\{E_{\rho}\right\} \subset \ell_{1}(S)$ is such that $\left\|E_{\rho}\right\| \leqq R$ for all $\rho$ and some positive number $R$, and in addition

$$
\left\|E_{\rho} * \delta_{x}-\delta_{x}\right\| \longrightarrow 0 \quad \text { for all } x \in S .
$$

Then $\left\{E_{\rho}\right\}$ is a bounded approximate identity for $\iota_{1}(S)$.

Proof. Let $\alpha \in \ell_{1}(S)$ be arbitrary and let $\varepsilon>0$ be given. Then there exists $N_{0}$ such that $\sum_{i=N_{0}+1}^{\infty}\left|\alpha\left(x_{i}\right)\right|<\varepsilon / 3 R$. Also, there exists $\rho_{0}$ such that $\rho \geqq \rho_{0}$ implies

$$
\left\|E_{\rho} * \delta_{x_{i}}-\delta_{x_{i}}\right\|<\frac{\varepsilon}{3 N_{0}\left|\alpha\left(x_{i}\right)\right|}, \quad i=1,2, \cdots, N_{0} .
$$

Thus,

$$
\begin{array}{rlrl}
\left\|E_{\rho} * \alpha-\alpha\right\| & \leqq\left\|\sum_{i=1}^{N_{0}} \alpha\left(x_{i}\right)\left(E_{\rho} * \delta_{x_{i}}-\delta_{x_{i}}\right)\right\| \\
& +\left\|E_{\rho} * \sum_{i=N_{0}+1}^{\infty} \alpha\left(x_{i}\right) \delta_{x_{i}}\right\|+\left\|\sum_{i=N_{0}+1}^{\infty} \alpha\left(x_{i}\right) \delta_{x_{i}}\right\| \\
& \leqq \frac{\varepsilon}{3}+\frac{\varepsilon}{3}+\frac{\varepsilon}{3}=\varepsilon & \text { for all } \rho \geqq \rho_{0} .
\end{array}
$$

This completes the proof.

We now confine our attention to separative semigroups. Using Proposition 4.1, we consider $S$ to be embedded in the compact 
topological semigroup $\Gamma$ as a dense subsemigroup.

It is possible to use the semicharacters of $S$ to decompose the semigroup into a set of equivalence classes as follows: for $x, y \in S$, write $x \sim y$ if for each $\chi \in \widehat{S}, \chi(x)=0$ if and only if $\chi(y)=0$; let $H_{x}=\{y \in S: y \sim x\}$; then $\sim$ is an equivalence relation on $S$ and the mapping $x \mapsto H_{x}$ is a homomorphism of $S$ onto the idempotent semigroup $\left\{H_{x}: x \in S\right\}$, multiplication in the latter space being given by $H_{x} H_{y}=H_{x y}$. Then define a partial ordering which makes $\left\{H_{x}: x \in S\right\}$ a semilattice by setting $H_{x} \leqq H_{y}$ if and only if $H_{x} H_{y}=H_{x}, x, y \in S$; further, define $A_{x}=\left\{y \in S: H_{y} \leqq H_{x}\right\}, x \in S$. Then for each $x \in S$, $H_{x}$ and $A_{x}$ are subsemigroups of $S ; H_{x}$ is cancellative;

$$
A_{x}=\{y \in S: \text { if } \chi \in \hat{S} \text { and } \chi(x) \neq 0 \text {, then } \chi(y) \neq 0\} \text {; }
$$

and the characteristic function of $A_{x}$ is always a semicharacter of $S$ (see $[2,3,5]$ for more details).

If $\ell_{1}(S)$ has a bounded approximate identity $\left\{E_{\rho}\right\}$, then certainly $\left\{E_{\rho}\right\}$ is a weak bounded approximate identity. Thus, Theorem 3.1 with $A=\ell_{1}(S)$ implies the existence of a finite set of relative units in $\Gamma$. In the next theorem we show that if we assume that $S$ has a set of relative units, then the existence of a finite set of relative units in $\Gamma$ allows us to construct a bounded approximate identity for $\ell_{1}(S)$.

THEOREM 4.5. Suppose $\ell_{1}(S)$ is semisimple and $S$ contains a set of relative units. If $\Gamma$ contains a finite set of relative units, then (a) there exist in $S$ a finite number of nets

$$
\left\{u_{\rho(i)}\right\}_{\rho(i) \in \mathscr{F}_{i}}, i=1,2, \cdots, n
$$

such that for every $x \in S$ there exist $j$ and $\rho(j)_{x}$ for which $\rho(j) \geqq \rho(j)_{x}$ implies $u_{\rho(j)} x=x$, and (b) $\ell_{1}(S)$ has a bounded (norm) approximate identity. Moreover, if $\Gamma$ has an identity, then $n=1$ in (a) and the approximate identity in (b) is of norm one.

Proof. Let $U=\left\{\gamma_{1}, \gamma_{2}, \cdots, \gamma_{n}\right\}$ be a set of relative units for $\Gamma$, and assume without loss of generality that $\gamma_{i}^{2}=\gamma_{i}$ for all $i$ [6]. Then there exist nets $\left\{x_{\rho(i)}\right\}_{\rho(i) \in \mathscr{F}_{i}} \subset S$ such that $x_{\rho(i)} \rightarrow \gamma_{i}, i=1,2, \cdots, n$. Let $\left\{u_{\rho(i)}\right\}_{\rho(i) \in F_{i}} \subset S$ be such that $u_{\rho_{(i)} x_{\rho(i)}}=x_{\rho_{(i)}}$ for all $\rho(i) \in \mathscr{F}_{i}, i=$ $1,2, \cdots, n$. Now, let $x \in S$; then there exists $j$ such that $\gamma_{j} x=x$. Thus, $x_{\rho(j)} x \rightarrow x$ in $\Gamma$. If $\phi_{x}$ is the characteristic function of $A_{x}, \phi_{x} \in \hat{S}$ [2, Proposition 3.8] and hence $\phi_{x}\left(x_{\rho(j)} x\right) \rightarrow \phi_{x}(x)=1$; thus, there exists $\rho(j)_{x}$ such that $\rho(j) \geqq \rho(j)_{x}$ implies $\phi_{x}\left(x_{\rho(j)}\right)=\phi_{x}\left(x_{\rho(j)} x\right)=1$. Hence, by the choice of $u_{\rho(j)}, \phi_{x}\left(u_{\rho(j)}\right)=1$ for all $\rho(j) \geqq \rho(j)_{x}$; we thus have 
that $\rho(j) \geqq \rho(j)_{x}$ implies $x_{\rho(j)} \in A_{x}, u_{\rho(j)} \in A_{x}$. We now show that $u_{\rho(j)} x=x$ for all $\rho(j) \geqq \rho(j)_{x}$. If not, there exist $\rho(j)$ and $\chi \in \hat{\Gamma}$ such that $\chi\left(u_{\rho(j)} x\right) \neq \chi(x)$. Since $u_{\rho(j)} x \in H_{x}$, if follows that $\left.\chi\left(u_{\rho(j)} x\right)\right) \neq 0$ and $\chi(x) \neq 0$. Also since $x_{\rho(j)} \in A_{x}, \chi\left(x_{\rho(j)}\right) \neq 0$ and since

$$
x_{\rho(j)} u_{\rho(j)}=x_{\rho(j)}, \chi\left(u_{\rho(j)}\right)=1 .
$$

However, this leads to the contradiction $\chi(x)=\chi\left(u_{\rho(j)} x\right) \neq \chi(x)$. Therefore, if $x \in S$ is arbitrary, then it is possible to find $j$ and $\rho(j)_{x}$ such that $\rho(j) \geqq \rho(j)_{x}$ implies $u_{\rho(j)} x=x$. This proves (a). To see that $\ell_{1}(S)$ has a bounded approximate identity, let

$$
\mathscr{F}=\mathscr{F}_{1} \times \mathscr{F}_{2} \times \cdots \times \mathscr{F}_{n}
$$

with $\rho \in \mathscr{F}$ denoted by $\rho=(\rho(1), \rho(2), \cdots, \rho(n))$. Further, for each $\rho \in \mathscr{F}$, define

$$
\begin{aligned}
E_{\rho}= & \sum_{1 \leqq i \leqq n} \delta_{u_{\rho(i)}}-\sum_{1 \leqq i<j \leqq n} \delta_{u_{\rho(i)} u_{\rho(j)}} \\
& +\cdots+(-1)^{n+1} \delta_{u_{\rho(1)} u_{\rho(2)} \ldots u_{\rho(n)}} .
\end{aligned}
$$

We assert that $\left\{E_{\rho}\right\}_{\rho \in \mathscr{F}}$ is a bounded approximate identity for $\iota_{1}(S)$. To substantiate this, let $x \in S$ and let $j$ and $\rho(j)_{x}$ be such that $\rho(j) \geqq$ $\rho(j)_{x}$ implies that $x u_{\rho(j)}=x$. Thus, for all $\rho \in \mathscr{F}$ with

$$
\rho=(\cdots, \rho(j), \cdots) \text { and } \rho(j) \geqq \rho(j)_{x},
$$

we have that $E_{\rho} * \delta_{x}=\delta_{x}$. Since $x$ is arbitrary, $E_{\rho} * \delta_{x} \rightarrow \delta_{x}$ for all $x \in S$ in $\ell_{1}$-norm. Clearly, $\left\|E_{\rho}\right\| \leqq R$ for some positive number $R$ and for all $\rho \in \mathscr{F}$. Thus, Proposition 4.4 implies that $\left\{E_{\rho}\right\}_{\rho \in \mathscr{F}}$ is a bounded approximate identity for $\ell_{1}(S)$. If $\Gamma$ has an identity, say $\gamma_{1}$, then $\left\{E_{\rho}\right\}$ with $E_{\rho}=\delta_{u_{\rho(1)}}$ is seen to be an approximate identity of norm one for $\ell_{1}(S)$.

Corollary 4.6. Suppose $\ell_{1}(S)$ is semisimple. Then $\ell_{1}(S)$ has a bounded approximate identity if and only if $S$ has a set of relative units and $\Gamma$ has a finite set of relative units. Moreover, $\ell_{1}(S)$ has a bounded approximate identity of norm one if and only if $S$ has a set of relative units and $\Gamma$ has an identity.

Proof. If $\ell_{1}(S)$ has a bounded approximate identity, then $S$ has a set of relative units by Proposition 4.2; $\Gamma$ contains a finite set of relative units by Theorem 3.1. Now if $\ell_{1}(S)$ has a bounded approximate identity of norm one, then $\Gamma$ has an identity by Corollary 3.2.

Conversely, if $S$ has a set of relative units and $\gamma$ has a finite set of relative units, then $\ell_{1}(S)$ has a bounded approximate identity by Theorem 4.5 (b). Moreover, if $\Gamma$ has an identity, then $\ell_{1}(S)$ has a 
bounded approximate identity of norm one by Theorem 4.5. This completes the proof.

Note the existence in $S$ of the nets described in Theorem 4.5 (a) is all that is required in order to construct a bounded approximate identity in $\ell_{1}(S)$.

Once again referring to Theorem 3.1 of [10], if $S$ is separative and we make the additional assumption that $S$ contains a set of relative units, then the existence of an identity in $\Gamma$ is a sufficient condition for $\ell_{1}(S)$ to have a bounded approximate identity of norm one by Corollary 4.6. Each of the next two results gives conditions on $S$ that are equivalent to the existence of a bounded approximate identity in $\ell_{1}(S)$. Theorem 4.8 is almost a restatement of Theorem 4.5 and Corollary 4.6.

Proposition 4.7. Suppose $\ell_{1}(S)$ is semisimple. Then $\ell_{1}(S)$ has a bounded approximate identity of norm one if and only if $S$ has a set of relative units and there exists a net $\left\{x_{\rho}\right\} \subset S$ such that $\chi\left(x_{\rho}\right) \rightarrow 1$ for all $\chi \in \widehat{S}$.

Proof. If $\ell_{1}(S)$ has a bounded approximate identity of norm one, then Corollary 4.6 implies that $S$ has a set of relative units and $\Gamma$ has an identity $e$. Assuming $S$ is embedded in $\Gamma$ as a dense subset, there exists a net $\left\{x_{\rho}\right\} \subset S$ such that $x_{\rho} \rightarrow e$. Hence, $\chi\left(x_{\rho}\right) \rightarrow \chi(e)=1$ for all $\chi \in \hat{S}$.

Conversely, if $S$ has a net $\left\{x_{\rho}\right\}$ such that $\chi\left(x_{\rho}\right) \rightarrow 1$ for all $\chi \in \hat{S}$, then $\left\{\delta_{x_{\rho}}\right\}$ is a weak bounded approximate identity of norm one. Thus, $\Gamma$ has an identity $e$ by Corollary 3.2. Since by assumption $S$ has a set of relative units, Corollary 4.6 implies that $\ell_{1}(S)$ has a bounded approximate identity of norm one.

THeOREM 4.8. Suppose $\ell_{1}(S)$ is semisimple. Then $\ell_{1}(S)$ has a bounded approximate identity if and only if there exist a finite number of nets $\left\{u_{\rho(i)}\right\}_{\rho(i) \in \mathscr{F}_{i}} \subset S, i=1,2, \cdots, n$ such that for every $x \in S$ there exist $j$ and $\rho(j)_{x}$ for which $\rho(j) \geqq \rho(j)_{x}$ implies $u_{\rho(j)} x=x$.

Proof. If $\ell_{1}(S)$ has a bounded approximate identity, then $S$ contains a set of relative units and $\Gamma$ contains a finite set of relative units by Corollary 4.6. Therefore, by Theorem 4.5 (a) there exist in $S$ the nets $\left\{u_{\rho(i)}\right\}_{\rho(i) \in \mathscr{F}_{i}}, \mathrm{i}=1,2, \cdots, n$ with the designated properties.

Conversely, if $S$ contains a finite number of nets $\left\{u_{\rho(i)}\right\}_{\rho(i) \in \mathscr{F}}$, $i=1,2, \cdots, n$ as specified in the statement of this theorem, then as in the proof of Theorem 4.5 (b), it is possible to construct a bounded approximate identity for $\ell_{1}(S)$. 
CoRollary 4.9. Suppose $\ell_{1}(S)$ is semisimple. Then $\ell_{1}(S)$ has a bounded approximate identity of norm one if and only if there is a net $\left\{u_{\rho}\right\} \subset S$ such that for every $x \in S$ there exists $\rho_{x}$ for which $\rho \geqq \rho_{x}$ implies $x u_{\rho}=x$. In this case $\left\{\delta_{u_{\rho}}\right\}$ forms a bounded approximate identity for $\iota_{1}(S)$.

Proof. Most of this corollary is obvious. In any event, the fact that $\left\|\delta_{u_{\rho}} * \delta_{x}-\delta_{x}\right\| \rightarrow 0$ for all $x \in S$ proves that $\left\{\delta_{u_{\rho}}\right\}$ forms a bounded approximate identity of norm one for $\ell_{1}(S)$ by Proposition 4.4. This completes the proof.

If $S$ is an idempotent semigroup, there is a natural partial ordering on $S$ that is sometimes helpful in constructing the net $\left\{u_{\rho}\right\}$ of Corollary 4.9. We define "ฏ” by $x \leqq y$ if $x y=x, x, y \in S$.

Proposition 4.10. Let $S$ be an idempotent semigroup. Then $\ell_{1}(S)$ has a bounded approximate identity of norm one if and only if $S$ has the property that for any $x, y \in S$ there exists $z \in S$ such that $x: z=x$ and $y z=y$.

Proof. Suppose $S$ has the designated property. Then the semigroup itself forms a net under the partial ordering described above, since by assumption, given $x, y \in S$ it is possible to find $z \in S$ such that $x \leqq z, y \leqq z$. Now, if $x \in S$, choose $z \in S$ such that $x \leqq z$. Then $y \in S, y \geqq z$ implies that $x y=x$ and so by Corollary $4.9\left\{\delta_{y}\right\}_{y \in S}$ forms an approximate identity for $\iota_{1}(S)$.

Conversely, if $\ell_{1}(S)$ has a bounded approximate identity of norm one, then by Corollary 4.9 there is a net $\left\{u_{\rho}\right\} \subset S$ such that given $x, y \in S$ it is possible to find $u_{\rho_{0}}$ with the property that $u_{\rho_{0}} x=x$ and $u_{\rho_{0}} y=y$. This completes the proof.

We would like to prove a theorem analogous to Proposition 4.10 for semigroups that are unions of groups, but not necessarily idempotent. Let $S^{\prime}=\left\{H_{x}: x \in S\right\}$; recall that under the multiplication $H_{x} H_{y}=H_{x y}, S^{\prime}$ is an idempotent semigroup [2,3].

Proposition 4.11. Suppose $\iota_{1}(S)$ is semisimple. Then $\ell_{1}(S)$ has a bounded approximate identity of norm one if and only if $S$ has a set of relative units and $\ell_{1}\left(S^{\prime}\right)$ has a bounded approximate identity of norm one.

Proof. The existence of a bounded approximate identity of norm one in $\ell_{1}(S)$ is equivalent to the existence of a net $\left\{u_{\rho}\right\}$ in $S$ with the property that for every $x \in S$ there exists $\rho_{x}$ such that $\rho \geqq \rho_{x}$ 
implies $x u_{\rho}=x$ by Corollary 4.9. Thus, $S$ has a set of relative units. Moreover, given $x, y \in S$, there exists $\rho_{0}, \rho_{0} \geqq \rho_{x}$ and $\rho_{0} \geqq \rho_{y}$, such that $u_{\rho_{0}} x=x$ and $u_{\rho_{0}} y=y$. Thus, $H_{x u_{\rho_{0}}}=H_{x}$ and $H_{y \rho_{0}}=H_{y}$. The idempotence of $S^{\prime}$ and Proposition 4.10 now imply that $\ell_{1}\left(S^{\prime}\right)$ has a bounded approximate identity of norm one.

Conversely, because $\ell_{1}\left(S^{\prime}\right)$ has a bounded approximate identity of norm one, Proposition 4.7 implies that there exists a net $\left\{H_{x_{\rho}}\right\} \subset S^{\prime}$ such that $\psi\left(H_{x_{\rho}}\right) \rightarrow 1$ for all $\psi \in\left(S^{\prime}\right)^{\wedge}$. Let $\left\{u_{\rho}\right\} \subset S$ be such that $u_{\rho} x_{\rho}=x_{\rho}$ for all $\rho$. We assert that $\chi\left(u_{\rho}\right) \rightarrow 1$ for all $\chi \in \hat{S}$. To substantiate this assertion, let $\chi \in \hat{S}$ and define for all $H_{x} \in S^{\prime}$,

$$
\tilde{\chi}\left(H_{x}\right)=\left\{\begin{array}{l}
1, \chi(x) \neq 0 \\
0, \chi(x)=0
\end{array}\right.
$$

Then $\tilde{\chi} \in\left(S^{\prime}\right)^{\wedge}$ and hence $\tilde{\chi}\left(H_{x_{\rho}}\right) \rightarrow 1$. Thus, there exists $\rho_{0}$ such that $\rho \geqq \rho_{0}$ implies that $\tilde{\chi}\left(H_{x_{\rho}}\right)=1$, which in turn implies that $\chi\left(x_{\rho}\right) \neq 0$ for all $\rho \geqq \rho_{0}$. Therefore, $\rho \geqq \rho_{0}$ implies that $\chi\left(u_{\rho}\right)=1$, or in other words, $\chi\left(u_{\rho}\right) \rightarrow 1$. Thus, an application of Proposition 4.7 yields that $\ell_{1}(S)$ has a bounded approximate identity of norm one.

THEOREM 4.12. Let $S$ be a union of disjoint groups. Then $\ell_{1}(S)$ has a bounded approximate identity of norm one if and only if $S$ has the property that corresponding to each $x, y \in S$ there is $z \in S$ such that $H_{x z}=H_{x}$ and $H_{y z}=H_{y}$.

Proof. The fact that $S$ is a union of disjoint groups is equivalent to $H_{x}$ being a group for all $x \in S$ [5, Theorem 8.11]. Thus, each $H_{x}$ contains an idempotent $u$ such that $u x=x$, and so $S$ has a set of relative units. If for each $x, y \in S$ there exists $z \in S$ such that $H_{x z}=H_{x}$ and $H_{y z}=H_{y}$, then $\ell_{1}\left(S^{\prime}\right)$ has a bounded approximate identity of norm one. Thus, according to Proposition $4.11 \ell_{1}(S)$ has a bounded approximate identity of norm one.

Conversely, if $\ell_{1}(S)$ has a bounded approximate identity of norm one, then it is an easy matter to see how to use the net described in Corollary 4.9 in order to obtain the property stated above. This completes the proof.

For the sake of completeness we state following proposition.

Proposition 4.13. Suppose $\ell_{1}(S)$ is semisimple. Then $\ell_{1}(S)$ has $a$ bounded approximate identity of norm one if and only if $S$ has a set of relative units and $\hat{S}$ is a semigroup.

Proof. If $\ell_{1}(S)$ has a bounded approximate identity of norm one, 
then $S$ has a set of relative units and $\Gamma$ has an identity by Corollary 4.6. Hence, $\hat{S}$ is a semigroup.

Conversely, if $\hat{S}$ is a semigroup, by Theorem 6.7 of [5], for each $x, y \in S$ there exists $z \in S$ such that $H_{x z}=H_{x}$ and $H_{y z}=H_{y}$. Thus, the idempotence of $S^{\prime}$ and Proposition 4.10 imply that $\ell_{1}\left(S^{\prime}\right)$ has a bounded approximate identity of norm one. Therefore, by Proposition 4.11 and the fact that $S$ has a set of relative units, we conclude that $\ell_{1}(S)$ has a bounded approximate identity of norm one. This completes the proof.

ExAMPLE 4.14. Let $S$ be the set of integers under the operation of maximum multiplication and consider the subsemigroup $S_{0}$ of $S \times S$ consisting of the negative axes

$$
\{(x, 0),(0, y): x, y \in S, x \leqq 0, y \leqq 0\} \text {. }
$$

Then, clearly, two sequences $\{(n, 0): n \leqq 0\}$ and $\{(0, m): m \leqq 0\}$ are required having the properties specified in Theorem 4.8; thus, by Theorem $4.8 \ell_{1}\left(S_{0}\right)$ has a bounded approximate identity. $\Gamma$ is the two point compactification of $S_{0}$ obtained by adjoining $\gamma_{1}$ and $\gamma_{2}$ and defining the products: $\gamma_{1}^{2}=\gamma_{1} ; \gamma_{2}^{2}=\gamma_{2} ; \gamma_{1} \gamma_{2}=(0,0) ; \gamma_{1}(n, 0)=(n, 0)$ for all $n \leqq 0 ; \gamma_{1}(0, m)=(0,0)$ for all $m \leqq 0 ; \gamma_{2}(0, m)=(0, m)$ for all $m \leqq 0$; and $\gamma_{2}(n, 0)=(0,0)$ for all $n \leqq 0 . \gamma_{1}$ and $\gamma_{2}$ are relative units for $\Gamma$ with $E=\delta_{\gamma_{1}}+\delta_{\gamma_{2}}-\delta_{(0,0)}$ being the Hewitt-Zuckerman identity for $M(\Gamma)$. If for $n \leqq 0, m \leqq 0$, we let $E_{(n, m)}=\delta_{(n, 0)}+\delta_{(0, m)}-\delta_{(0,0)}$, then $\left\{E_{(n, m)}\right\}$ is a bounded approximate identity for $\iota_{1}(S)$.

\section{REFERENCES}

1. R. Arens, Operations induced in function classes, Monatsh. für Math., 55 (1951), 1-19.

2. W. W. Comfort, The isolated points in the dual of a commutative semigroup, Proc. Amer. Math. Soc., 11 (1960), 227-233.

3. - The Silov boundary induced by a certain Banach algebra, Trans. Amer. Math. Soc., 98 (1961), 501-517.

4. I. Glicksberg, Convolution semigroups of measures, Pacific J. Math., 9 (1959), 51-67.

5. E. Hewitt and H. S. Zuckerman, The $\ell_{1}$-algebra of a commutative semigroup, Trans. Amer. Math. Soc., 83 (1956), 70-97.

6. L. J. Lardy, On the identity in a measure algebra, Proc. Amer. Math. Soc., 19 (1968), 807-810.

7. M. Petrich, The translation hull in semigroups and rings, Semigroup Forum, 1 (1970), 283-360.

8. J. F. Rennison, Arens products and measure algebras, J. London Math. Soc., 44 (1969), 369-377.

9. C. E. Rickart, General Theory of Banach Algebras, Van Nostrand Co., Princeton . London · New York, 1960.

10. J. L. Taylor, The structure of convolution measure algebras, Trans. Amer. Math. Soc., 119 (1965), 150-166. 
Received March 27, 1972. The author wishes to express his sincere thanks to Professor Lawrence J. Lardy, without whose help this paper could not have been completed. The results presented here comprise a portion of the author's doctoral dissertation written while in attendance at Syracuse University, Syracuse, New York.

Bell telephone Laboratories, INC. 



\section{PACIFIC JOURNAL OF MATHEMATICS}

\section{EDITORS}

D. Gilbarg and J. Milgram

Stanford University

Stanford, California 94305

R. A. Beaumont

University of Washington

Seattle, Washington 98105
J. DUGUNDJI* Department of Mathematics

University of Southern California Los Angeles, California 90007

RICHARD ARENS

University of California

Los Angeles, California 90024

\section{ASSOCIATE EDITORS}

E. F. BeCKenbach

B. H. NeUMaNN

F. WOLF

K. Yoshida

\section{SUPPORTING INSTITUTIONS}

UNIVERSITY OF BRITISH COLUMBIA

UNIVERSITY OF SOUTHERN CALIFORNIA

CALIFORNIA INSTITUTE OF TECHNOLOGY

UNIVERSITY OF CALIFORNIA

MONTANA STATE UNIVERSITY

STANFORD UNIVERSITY

UNIVERSITY OF TOKYO

UNIVERSITY OF NEVADA

UNIVERSITY OF UTAH

NEW MEXICO STATE UNIVERSITY

WASHINGTON STATE UNIVERSITY

OREGON STATE UNIVERSITY

UNIVERSITY OF OREGON

OSAKA UNIVERSITY

UNIVERSITY OF WASHINGTON

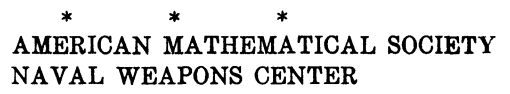

* C. DePrima will replace J. Dugundji until August 1974. 


\section{Pacific Journal of Mathematics}

\section{Vol. 47, No. $1 \quad$ January, 1973}

K. Adachi, Masuo Suzuki and M. Yoshida, Continuation of holomorphic

mappings, with values in a complex Lie group ....................

Michael Aschbacher, A characterization of the unitary and symplectic groups

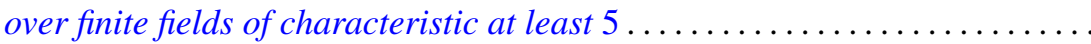

Larry Eugene Bobisud and James Calvert, Energy bounds and virial theorems for abstract wave equations....................................

Christer Borell, A note on an inequality for rearrangements ................

Peter Southcott Bullen and S. N. Mukhopadhyay, Peano derivatives and general

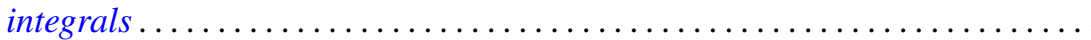

Wendell Dan Curtis, Yu-Lee Lee and Forrest Miller, A class of infinite dimensional subgroups of $\operatorname{Diff}^{r}(X)$ which are Banach Lie groups .........

Paul C. Eklof, The structure of ultraproducts of abelian groups ...............

William Alan Feldman, Axioms of countability and the algebra $C(X) \ldots \ldots \ldots$

Jack Tilden Goodykoontz, Jr., Aposyndetic properties of hyperspaces...........

George Grätzer and J. Płonka, On the number of polynomials of an idempotent algebra. II ...........................................

Alan Trinler Huckleberry, The weak envelope of holomorphy for algebras of

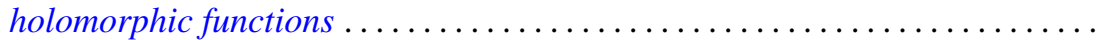

John Joseph Hutchinson and Julius Martin Zelmanowitz, Subdirect sum decompositions of endomorphism rings . . . . . . . . . . . . . . . .

Gary Douglas Jones, An asymptotic property of solutions of

$y^{\prime \prime \prime}+p y^{\prime}+q y=0$.

Howard E. Lacey, On the classification of Lindenstrauss spaces .

Charles Dwight Lahr, Approximate identities for convolution measure algebras.

George William Luna, Subdifferentials of convex functions on Banach

spaces.

Nelson Groh Markley, Locally circular minimal sets. .

Robert Wilmer Miller, Endomorphism rings of finitely generated projective modules

Donald Steven Passman, On the semisimplicity of group rings of linear

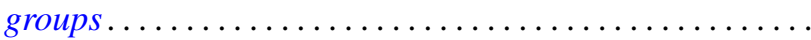

Bennie Jake Pearson, Dendritic compactifications of certain dendritic spaces.

Ryōtarō Satō, Abel-ergodic theorems for subsequences ...... .

Henry S. Sharp, Jr., Locally complete graphs. . .

Harris Samuel Shultz, A very weak topology for the Mikusinski field of

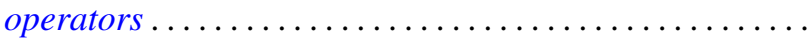

Elena Stroescu, Isometric dilations of contractions on Banach spaces ...

Charles W. Trigg, Versum sequences in the binary system ... . .

William L. Voxman, On the countable union of cellular decompositions of n-manifolds 